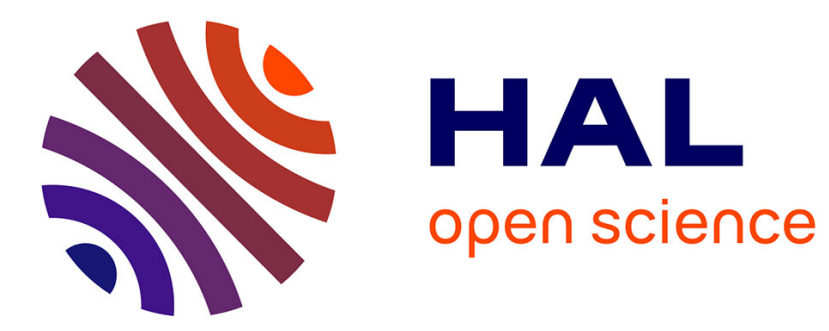

\title{
Loci of extrema of thermodynamic response functions for the Lennard-Jones fluid
}

Claudio Cerdeiriña, Pablo Téllez-Arredondo, Milton Medeiros, Manuel Piñeiro

\section{To cite this version:}

Claudio Cerdeiriña, Pablo Téllez-Arredondo, Milton Medeiros, Manuel Piñeiro. Loci of extrema of thermodynamic response functions for the Lennard-Jones fluid. Molecular Physics, 2011, 109 (20), pp.2443-2449. 10.1080/00268976.2011.619505 . hal-00743045

\section{HAL Id: hal-00743045 \\ https://hal.science/hal-00743045}

Submitted on 18 Oct 2012

HAL is a multi-disciplinary open access archive for the deposit and dissemination of scientific research documents, whether they are published or not. The documents may come from teaching and research institutions in France or abroad, or from public or private research centers.
L'archive ouverte pluridisciplinaire HAL, est destinée au dépôt et à la diffusion de documents scientifiques de niveau recherche, publiés ou non, émanant des établissements d'enseignement et de recherche français ou étrangers, des laboratoires publics ou privés. 


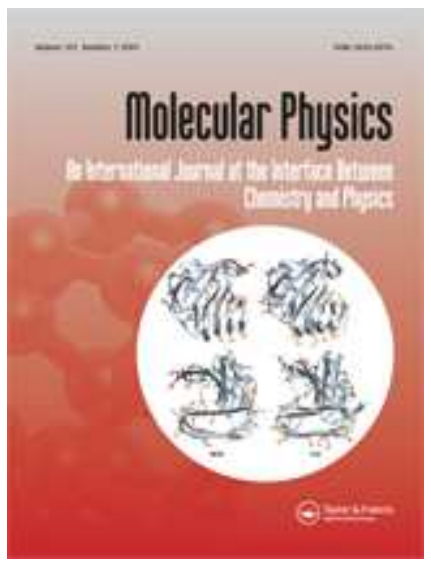

\section{Loci of extrema of thermodynamic response functions for the Lennard-Jones fluid}

\begin{tabular}{|c|c|}
\hline Journal: & Molecular Physics \\
\hline Manuscript ID: & TMPH-2011-0267.R1 \\
\hline Manuscript Type: & Full Paper \\
\hline $\begin{array}{r}\text { Date Submitted by the } \\
\text { Author: }\end{array}$ & 21-Aug-2011 \\
\hline Complete List of Authors: & $\begin{array}{l}\text { Cerdeiriña, Claudio; Universidad de Vigo, Departamento de Física } \\
\text { Aplicada } \\
\text { Téllez-Arredondo, Pablo; Universidad Nacional Autónoma de } \\
\text { México, Departamento de Físicoquímica } \\
\text { Medeiros, Milton; Universidad Nacional Autónoma de México, } \\
\text { Departamento de Físicoquímica } \\
\text { Piñeiro, Manuel; Universidad de Vigo, Departamento de Física } \\
\text { Aplicada }\end{array}$ \\
\hline Keywords: & $\begin{array}{l}\text { thermodynamic response functions, loci of extrema, Lennard-Jones } \\
\text { fluid }\end{array}$ \\
\hline \multicolumn{2}{|c|}{$\begin{array}{l}\text { Note: The following files were submitted by the author for peer review, but cannot be converted } \\
\text { to PDF. You must view these files (e.g. movies) online. }\end{array}$} \\
\hline Source files.zip & \\
\hline
\end{tabular}

\section{SCHOLARONE \\ Manuscripts}




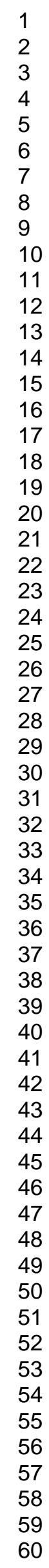

URL: http://mc.manuscriptcentral.com/tandf/tmph 


\title{
FULL PAPER
}

\section{Loci of extrema of thermodynamic response functions}

\section{for the Lennard-Jones fluid}

\begin{abstract}
${ }^{a}$ Departamento de Fisicoquímica, Facultad de Química, Universidad Nacional Autónoma de México, Ciudad de México 04510, Mexico

${ }^{\mathrm{b}}$ Departamento de Física Aplicada, Universidad de Vigo - Campus del Mar, As Lagoas s/n, 32004 Ourense, Spain
\end{abstract}




\title{
Loci of extrema of thermodynamic response functions \\ for the Lennard-Jones fluid
}

\begin{abstract}
Lines of extrema along isotherms and isobars for the residual isochoric heat capacity, the residual isobaric heat capacity, the isobaric thermal expansivity, and the isothermal compressibility of the Lennard-Jones fluid have been studied from popular equations of state due to Johnson et al. [Mol. Phys. 78, 591 (1993)], Kolafa and Nezbeda [Fluid Phase Equilib. 100, 1 (1994)], and Mecke et al. [Int. J. Thermophys. 17, 391 (1996)]. On depicting such loci in the pressure-temperature plane, the characteristic behavior of thermodynamic response functions in the ideal-gas limit (at high enough temperatures or low enough pressures), the close-packed-fluid limit (at low enough temperatures or high enough pressures) as well as in the liquid and critical regions is identified. The present analysis is informative itself, but it also stimulates further work in order to tackle more complicated cases of study including associated fluids like alcohols and low-temperature water.
\end{abstract}

Keywords: thermodynamic response functions; loci of extrema; Lennard-Jones fluid 


\section{Introduction}

In 1965 Rowlinson asserted [1] that the thermodynamic properties of the fluid phases of simple substances can be conveniently mapped by considering the lines in $p-V$ $T$ space along which important derivatives vanish. He exposed the experimental situation for the so-called Brown characteristic curves [2], namely, the Amagat (or Joule inversion), Boyle, and Charles (or Joule-Thomson inversion) lines, defined, respectively, by Equations (1) to (3) below:

$(\partial Z / \partial T)_{V}=0$

$(\partial \mathrm{Z} / \partial V)_{T}=0$

$(\partial Z / \partial T)_{p}=0$,

where $Z \equiv p V / R T$ is the compressibility factor. Two further lines of second-order derivatives or thermodynamic response functions were mentioned: from

$\left(\partial C_{V}^{r e s} / \partial V\right)_{T}=0 \Leftrightarrow\left(\partial C_{V}^{r e s} / \partial p\right)_{T}=0$

$\left(\partial C_{p}^{r e s} / \partial V\right)_{T}=0 \Leftrightarrow\left(\partial C_{p}^{r e s} / \partial p\right)_{T}=0$,

one gets loci of extrema for the residual isochoric and isobaric heat capacities, $C_{V}^{r e s} \equiv C_{V}-C_{V}^{i g}$ and $C_{p}^{r e s} \equiv C_{p}-C_{p}^{i g}$ (superscript "ig" denotes property in the ideal-gas state), along a path of constant temperature, that is, they are loci of isothermal extrema.

As it has been emphasized recently in connection with the Amagat line [3], calculation of the above loci is highly demanding for equations of state (EoS). Moreover, since adequate representation of thermodynamic response functions constitutes a stringent test for EoS [4], it may not be surprising that much care must be taken when 
dealing with loci defined by Equations (4) and (5). Such a task was undertaken by Stephenson [5-14], who, in addition to Brown's characteristic curves and the line defined by Equation (4), studied the loci of isobaric extrema of the isobaric thermal expansivity $\alpha_{p}$, given by

$\left(\partial \alpha_{p} / \partial V\right)_{p}=0 \Leftrightarrow\left(\partial \alpha_{p} / \partial T\right)_{p}=0$

Here we report calculations of the isothermal and isobaric lines of extrema for $C_{V}^{r e s}, C_{p}^{r e s}, \alpha_{p}$, and for the isothermal compressibility $\kappa_{T}$ of the Lennard-Jones fluid. To this end, the widely known EoS by Johnson et al. [15], Kolafa and Nezbeda [16], and Mecke et al. [17] were employed. Our results complement those by Stephenson on two counts: (i) they have been obtained on the solid basis of correlating EoS for a complete set of simulation data for the Lennard-Jones fluid and (ii) the study is extended to unexplored-to-date lines, defined from

$\left(\partial C_{V}^{r e s} / \partial V\right)_{p}=0 \Leftrightarrow\left(\partial C_{V}^{r e s} / \partial T\right)_{p}=0$,

$\left(\partial C_{p}^{r e s} / \partial V\right)_{p}=0 \Leftrightarrow\left(\partial C_{p}^{r e s} / \partial T\right)_{p}=0$

$\left(\partial \alpha_{p} / \partial V\right)_{T}=0 \Leftrightarrow\left(\partial \alpha_{p} / \partial p\right)_{T}=0$,

$\left(\partial \kappa_{T} / \partial V\right)_{T}=0 \Leftrightarrow\left(\partial \kappa_{T} / \partial p\right)_{T}=0$,

$\left(\partial \kappa_{T} / \partial V\right)_{p}=0 \Leftrightarrow\left(\partial \kappa_{T} / \partial T\right)_{p}=0$.

Results, as graphically represented in the regions of the $p-T$ plane corresponding to the stable fluid phases, are found to be relevant themselves, but also with a view to extend calculations to associated fluids including the study of water at low temperatures. 


\section{Equations of state for the Lennard-Jones fluid}

The three equations of state employed provide an expression for the residual Helmholtz energy $A_{\text {res }}$ as a function of temperature $T$ and number density $\rho \equiv N / V(N$ is the number of particles). For convenience, variables will be made dimensionless: $T^{*} \equiv T k_{\mathrm{B}} / \varepsilon, \rho^{*} \equiv \rho \sigma^{3}, p^{*} \equiv p \sigma^{3} / \varepsilon$, and $A_{r e s}^{*}=A_{r e s} / N \varepsilon$, where $\varepsilon$ and $\sigma$ are the LJ characteristic parameters (well depth and atomic diameter) while $k_{B}$ is the Boltzmann constant.

In 1993 Johnson et al. [15] presented a comprehensive set of simulation data for the LJ fluid and used them to improve a modified Benedict-Webb-Rubin (MBWR) equation of state, previously used by Nicolas et al. [18]. The corresponding residual Helmholtz energy for this MBWR EoS, which has 33 adjustable parameters, is given by:

$$
A_{\text {res }}^{*}=\sum_{i=1}^{8} \frac{a_{i}\left(T^{*}\right) \rho^{* i}}{i}+\sum_{i=1}^{6} b_{i}\left(T^{*}\right) G_{i}\left(\rho^{*}\right)
$$

The explicit functional forms of $a_{i}\left(T^{*}\right), b_{i}\left(T^{*}\right)$, and $G_{i}\left(\rho^{*}\right)$ can be found in the original paper. This equation provides an accurate correlation of pressure and internal energy from the triple point to $4.5 T_{c}$ (where $T_{c}$ denotes the critical temperature).

A year later, in 1994, Kolafa and Nezbeda [16] developed a 20-parameter EoS based on a perturbed virial expansion (PVE) with a theoretically defined reference hard sphere term. They employed the hybrid Barker-Henderson (hBH) perturbation theory, giving rise to the following expression for the residual Helmholtz energy:

$$
A_{r e s}^{*}=A_{H S}^{*}+\exp \left(-\gamma \rho^{* 2}\right) \rho^{*} T^{*} \Delta B_{2, h B H}+\sum_{i j} C_{i j} T^{* i / 2} \rho^{* j}
$$

where 


$$
\begin{gathered}
A_{H S}^{*}=T^{*}\left[\frac{5}{3} \ln (1-\eta)+\frac{\eta\left(34-33 \eta+4 \eta^{2}\right)}{6(1-\eta)^{2}}\right], \\
\eta=\frac{\pi \rho^{*} d_{h B H}^{3}}{6} \\
d_{h B H}=\sum_{i} C_{i} T^{*} \frac{i}{2}+C_{l n} \ln \left(T^{*}\right), \\
\Delta B_{2, h B H}=\sum_{i} C_{i} T^{* i / 2} .
\end{gathered}
$$

The first two terms in Equation (13) correspond to the perturbed virial expansion while the last one is a small empirical correction term, $A_{H S}^{*}$ is the hard sphere Helmholtz energy from Boublík and Nezbeda [19], $\eta$ is the packing fraction, $d_{h B H}$ is the temperaturedependent hard sphere diameter, $\Delta B_{2, h B H}$ is the residual second virial coefficient, and $i$ and $j$ are specific exponents. Values for the (constant) coefficients $\gamma, C_{i j}, C_{i}, C_{l n}$, and the exponents $i$ and $j$ can be found in the original paper. Kolafa and Nezbeda's EoS, termed $\mathrm{PVE} / \mathrm{hBH}$, can be employed from the triple point up to $7 T_{c}$. It provides very accurate predictions for compressibility, internal energy, chemical potential, second virial coefficient, and properties at vapor-liquid coexistence.

Then, in 1996 Mecke et al. [17] proposed a generalized Van der Waals-type equation of state, henceforth to be referred to as VdW-LJ, which uses 32 adjustable parameters. Concretely:

$$
\begin{aligned}
A_{r e s}^{*}=A_{H S^{\prime}}^{*} & +A_{A D F}^{*} \\
= & T^{*} \frac{4 \xi-3 \xi^{2}}{(1-\xi)^{2}}+T^{*} \sum_{i} c_{i}\left(T^{*} / T_{c}^{*}\right)^{m_{i}}\left(\rho^{*} / \rho_{c}^{*}\right)^{n_{i}} \exp \left[p_{i}\left(\rho^{*} / \rho_{c}^{*}\right)^{q_{i}}\right],
\end{aligned}
$$

where $A_{H S}^{*}$ is the hard sphere Helmholtz energy due to Carnahan and Starling [20], $\xi$ is the packing fraction from Saager et al. [21] correlation $\left(\xi=0.1617\left(\rho^{*} / \rho_{c}^{*}\right)[0.689+\right.$ $\left.\left.0.311\left(T^{*} / T_{c}^{*}\right)^{0.3674}\right]^{-1}\right), A_{A D F}^{*}$ is the Helmholtz energy of the attractive dispersive forces, 
$T_{c}^{*}$ and $\rho_{c}^{*}$ are the dimensionless critical temperature and density, respectively, and $m_{i}$, $n_{i}, p_{i}, q_{i}$ and $c_{i}$ are adjustable parameters (see Refs. 17 and 22). This EoS covers the whole fluid region up to the highest densities from the triple point up to $T^{*}=10$, producing very accurate results for pressure, internal energy, second and third virial coefficients, enthalpy of vaporization and properties at vapor-liquid coexistence.

\section{Calculation of loci of extrema}

To get explicit results, we first obtain the pressure from $p=-(\partial A / \partial V)_{T, N}$, where $A=A_{i g}+A_{\text {res }}$. In dimensionless form,

$$
p^{*}=\rho^{*} T^{*}+\rho^{* 2}\left(\frac{\partial A_{r e s}^{*}}{\partial \rho^{*}}\right)_{T^{*}}
$$

Thermodynamic derivatives are determined using standard thermodynamic relations. Hence, for the dimensionless isothermal compressibility $\kappa_{T}^{*} \equiv \kappa_{T} \varepsilon / \sigma^{3}$ and isobaric thermal expansivity $\alpha_{p}^{*} \equiv \alpha_{p} \varepsilon / k_{B}$ we find

$$
\begin{aligned}
& \kappa_{T}^{*}=\frac{1}{\rho^{*}}\left(\frac{\partial p^{*}}{\partial \rho^{*}}\right)_{T^{*}}^{-1}, \\
& \alpha_{p}^{*}=\left(\frac{\partial p^{*}}{\partial T^{*}}\right)_{\rho^{*}} \kappa_{T}^{*}
\end{aligned}
$$

The dimensionless isochoric residual heat capacity per particle $C_{V}^{r e s^{*}} \equiv C_{V}^{\text {res }} / N k_{B}$ is obtained via

$$
C_{V}^{\text {res* }}=-T^{*}\left(\frac{\partial^{2} A_{r e s}^{*}}{\partial T^{* 2}}\right)_{\rho^{*}},
$$




\section{Results and discussion}

Figures 1 to 4 show all studied loci in the $p-T$ (or, more precisely, $p^{*}-T^{*}$ ) plane. The three equations of state perform similarly for $C_{V}^{\text {res }}$ and $\kappa_{T}$ while important differences are observed for loci of isobaric extrema of $\alpha_{p}$ and isothermal and isobaric extrema of $C_{p}^{r e s}$. Results from $\mathrm{PVE} / \mathrm{hBH}$ for the two former lines are similar to those reported by Stephenson (see, e.g., Ref. 5). This fact leads one to ascribe their nonregular, strange shapes as obtained from MBWR and vdW-LJ to numerical pitfalls. On recalling that all equations of state behave well for $C_{V}^{\text {res }}$ and $\kappa_{T}$, one concludes that problems for the these three types of lines originate from $\alpha_{p}$. We must note that the situation for thermodynamic response functions contrasts with findings by Boshkova and Deiters for 
the Amagat line, which evidence that vdW-LJ shows the best performance at low temperatures.

Symbols + and - in plots indicate the sign of derivatives with respect to pressure in the plots of isothermal extrema [(a) panels] or with respect to temperature in the plots of isobaric extrema [(b) panels]. Specifically, $\left(\partial C_{V}^{\text {res }} / \partial p\right)_{T}$ is negative inside the closed loops of Figure 1a, which correspond to isothermal extrema (maxima or minima) of $C_{V}^{\text {res }}$, and positive outside them. Analogously, loci of isobaric extrema of $C_{V}^{\text {res }}$ in Figure $1 \mathrm{~b}$ enclose regions of positive $\left(\partial C_{V}^{\text {res }} / \partial T\right)_{p}$ [with $\left(\partial C_{V}^{\text {res }} / \partial T\right)_{p}<0$ outside the loops]. Consequent conclusions are obtained for $\left(\partial C_{p}^{r e s} / \partial p\right)_{T}$ in Figure $2 \mathrm{a},\left(\partial C_{p}^{r e s} / \partial T\right)_{p}$ in Figure 2b, $\left(\partial \alpha_{p} / \partial T\right)_{p}$ in Figure 3b, and $\left(\partial \kappa_{T} / \partial p\right)_{T}$ in Figure 4a. On the other hand, $\alpha_{p}$ just exhibits a locus of isothermal maxima, with $\left(\partial \alpha_{p} / \partial p\right)_{T}$ being positive below such line and negative in the remaining regions of the $p-T$ plane (see Figure 3a). And $\left(\partial \kappa_{T} / \partial T\right)_{p}$ is negative below lines of extrema in Figure $4 \mathrm{~b}$ and positive otherwise. Clearly, to visualize the behavior of response functions, plots of isothermal extrema [(a) panels] must be seen "vertically" whereas those of isobaric extrema [(b) panels] must be analyzed "horizontally". That is, on following, by way of example, a (super) near-critical isotherm in Figure 1a, one realizes that $C_{V}^{\text {res }}$ increases with $p$ at sufficiently high and low pressures and exhibits a maximum followed by a minimum in between; or, along a (super) near-critical isobar in Figure $1 \mathrm{~b}$, it is found that $C_{V}^{\text {res }}$ decreases with $T$ at sufficiently high and low temperatures and shows a minimum followed by a maximum in between. 
At low enough pressures or high enough temperatures, the ideal-gas limit, namely, $C_{V}^{r e s}, C_{p}^{r e s} \rightarrow 0, \alpha_{p} \rightarrow 1 / T$, and $\kappa_{T} \rightarrow 1 / p$, is recovered. At high enough pressures or low enough temperatures, the structure of the system may correspond to that of a close-packed fluid. In such regions, $C_{p}^{r e s}$ and $C_{V}^{r e s}$ decrease with $T$ and increase with $p, \alpha_{p}$ decreases with $T$ and $p$, and $\kappa_{T}$ increases with $T$ and decreases with $p$. In some cases, the expected behavior at low temperatures and pressures is not seen for the stable liquid because of crystallization (see, e.g., the plot of isobaric extrema of $\alpha_{p}$ ).

The pattern of behavior in the close-packed-fluid limit has been observed for molecular liquids at high pressures (primarily for $\alpha_{p}(T, p)$ data [25]) and ionic liquids at room temperature and atmospheric pressure [26-28]. That ionic liquids display wide liquid ranges with high critical temperatures [29] explains the observation of negative $\left(\partial \alpha_{p} / \partial T\right)_{p}$ and positive $\left(\partial C_{p}^{r e s} / \partial p\right)_{T}$ at ambient conditions [26]. Understandably, fluids of markedly different physicochemical nature behave similarly near close packing. The present results therefore cover behaviors beyond the LJ fluid.

In the liquid range, say, at subcritical pressures and temperatures not very far from the critical one so that fluctuations are non-negligible, $C_{V}^{\text {res }}, C_{p}^{\text {res }}, \alpha_{p}$, and $\kappa_{T}$ increase with $T$ and decrease with $p$. Even in the case that temperature is far below $T_{c}$ so that $\left(\partial C_{V}^{r e s} / \partial T\right)_{p}$ and $\left(\partial C_{p}^{\text {res }} / \partial T\right)_{p}$ are negative, $C_{V}=C_{V}^{i g}+C_{V}^{\text {res }}$ and $C_{p}=C_{p}^{i g}+C_{p}^{\text {res }}$ normally increase with $T$ because $C_{V}^{i g}$ and $C_{p}^{i g}$ are increasing functions of $T$ that usually dominate over the residual contribution. 
At supercritical isobars or supercritical isotherms all properties show maxima that belong to loci emanating from the vapor-liquid critical point, where $C_{V}^{\text {res }}, C_{p}^{\text {res }}, \alpha_{p}$, and $\kappa_{T}$ diverge. In fact, though not shown here explicitly, asymptotically close to criticality all loci converge at the so-called Widom line (namely, the line of maxima of the correlation length), which has received considerable attention recently in connection with water's hypothetical second (liquid-liquid) critical point [30]. It is important to note, however, that maxima in thermodynamic response functions do not necessarily mark the proximity of criticality. Simulations on systems with no critical points, like onedimensional fluids interacting via short-range pairwise interactions (including LennardJones intermolecular potentials), reveal maxima in $C_{V}^{\text {res }}$ [31]. As discussed previously $[31,32]$, in going from the close-packed-fluid limit to the ideal-gas limit, energy fluctuations — which according to statistical mechanics contribute positively to $C_{V}^{\text {res }}$ inevitably result in $C_{V}^{\text {res }}(T)$ and $C_{V}^{r e s}(p)$ maxima.

\section{Further remarks and outlook}

We have shown that the analysis of loci of extrema of thermodynamic response funcions in the $p-T$ plane is an adequate way of studying the global behavior of such properties. Results for the Lennard-Jones fluid may serve as a reference point: in view of what is known from experiments, we are led to think that it also qualitatively represents the behavior of many nonassociated molecular organic fluids, in accord with what Troncoso et al. [25] have recently found from their study for loci of extrema of $\alpha_{p}$. 
Association via hydrogen bonding gives rise to additional extrema. Indeed, it has been shown [33] that energetic effects of hydrogen bonding in alcohols can give rise to $C_{V}^{r e s}(T)$ and $C_{p}^{r e s}(T)$ maxima at atmospheric pressure and subcritical temperatures. Therefore, at a (super) near-critical isobar $C_{V}^{\text {res }}$ vs $T$ and $C_{p}^{\text {res }}$ vs $T$ curves exhibiting two maxima are expected for these fluids. And in certain specific cases such behavior can also be found for total heat capacities: a $C_{p}(T)$ maximum arising from association effects has been observed experimentally for branched alcohols [33,34]. It seems therefore natural to extend the present analysis to associated fluids with the aid of specific EoS (see, for instance, Refs. 35-39).

Water represents a case of study of extreme complexity because of its anomalous thermodynamics at low temperatures, down to the deeply supercooled regime [40]. The hypothetical existence of a liquid-liquid critical point in such region [40] may result in heat-capacity and $\kappa_{T}$ maxima as well as in $\alpha_{p}$ minima (since $\alpha_{p}$ is expected to diverge to $-\infty$ at that critical point). Even in the so-called singularity-free scenario [41], where anomalies are explained without any appeal to a second critical point, loci of extrema are present $[41,42]$. Certainly, tracing out loci of extrema for water's EoS should reveal the richest phenomenology among common organic fluids.

\section{Acknowledgements}

Financial support from "Consellería de Educación e Ordenación Universitaria (Xunta de Galicia)" and "Ministerio de Ciencia e Innovación" of Spain (\#FIS2009-07923) is gratefully acknowledged. Research of P.T-A. was supported by "Consejo Nacional de Ciencia y Tecnología CONACyT” of Mexico. 


\section{References}

[1] J.S. Rowlinson, Rep. Prog. Phys. 28, 169 (1965).

[2] E.H. Brown, Bulletin Inst. Ind. de Froid Annexe (International Institute of Refrigeration, 1960-1), pp. 169.

[3] O.L. Boshkova and U.K. Deiters, Int. J. Thermophys. 31, 227 (2010).

[4] J. Gregorowicz, J.P. O’Connell, and C.J. Peters, Fluid Phase Equilib. 116, 94 (1996).

[5] J. Stephenson, Phys. Chem. Liq. 8, 235 (1979).

[6] J. Stephenson and H.K. Leung, Phys. Chem. Liq. 8, 249 (1979).

[7] J. Stephenson, Phys. Chem. Liq. 8, 265 (1979).

[8] J. Stephenson, Phys. Chem. Liq. 9, 23 (1979).

[9] J. Stephenson, Phys. Chem. Liq. 9, 37 (1979).

[10] J. Stephenson, Phys. Chem. Liq. 9, 51 (1979).

[11] J. Stephenson, Phys. Chem. Liq. 9, 175 (1980).

[12] J. Stephenson, Phys. Chem. Liq. 10, 153 (1980).

[13] J. Stephenson and R. Couzens, Phys. Chem. Liq. 10, 167 (1980).

[14] J. Stephenson, Phys. Chem. Liq. 10, 229 (1981).

[15] J.K. Johnson, J.A. Zollweg, and K.E. Gubbins, Mol. Phys. 78, 591 (1993).

[16] J. Kolafa and I. Nezbeda, Fluid Phase Equilib. 100, 1 (1994).

[17] M. Mecke, A. Müller, J. Winkelmann, J. Vrabec, J. Fischer, R. Span, and W. Wagner, Int. J. Thermophys. 17, 391 (1996).

[18] J.J. Nicolas, K.E. Gubbins, W.B. Streett, and D.J. Tildesley, Mol. Phys. 37, 1429 (1979). 
[19] T. Boublík and I. Nezbeda, Collec. Czech. Chem. Commun. 51, 2301 (1986).

[20] N.F. Carnahan and K.E. Starling, J. Chem. Phys. 51, 635 (1969).

[21] B. Saager, R. Hennenberg, and J. Fischer, Fluid Phase Equilib. 72, 41 (1992).

[22] M. Mecke, A. Müller, J. Winkelmann, J. Vrabec, J. Fischer, R. Span, and W. Wagner, Int. J. Thermophys. 19, 1493 (1998).

[23] W.H. Press, S.A. Teulolsky, W.T., Vetterling, B.P. Flannery, Numerical Recipes in Fortran 77, second edition (Cambridge University Press, Cambridge, UK, 1992), pp. 180184.

[24] E.A. Mastny and J.J. de Pablo, J. Chem. Phys. 127, 104504 (2007).

[25] J. Troncoso, P. Navia, L. Romaní, D. Bessieres, and T. Lafitte, J. Chem. Phys. 134, 097502 (2011).

[26] J. Troncoso, C.A. Cerdeiriña, P. Navia, Y.A. Sanmamed, D. González-Salgado, and L. Romaní, J. Phys. Chem. Lett. 1, 211 (2010).

[27] P. Navia, J. Troncoso, and L. Romaní, J. Chem. Eng. Data 55, 595 (2010).

[28] Y.A. Sanmamed, P. Navia, D. González-Salgado, J. Troncoso, and L. Romaní, J. Chem. Eng. Data 55, 600 (2010).

[29] L.P.N. Rebelo, J.N.C. Lopes, J.M.S.S. Esperança, and E. Filipe, J. Phys. Chem. B 109, 6040 (2005).

[30] G. Franzese and H.E. Stanley, J. Phys.: Condens. Matter 19, 205126 (2007).

[31] B.C. Freasier, C.E. Woodward, and R.J. Bearman, J. Chem. Phys. 106, 10318 (1997).

[32] M.M. Piñeiro, C.A. Cerdeiriña, and M. Medeiros, J. Chem. Phys. 129, 014511 (2008). 
[33] C.A. Cerdeiriña, D. González-Salgado, L. Romaní, M.C. Delgado, L.A. Torres, and M. Costas, J. Chem. Phys. 120, 6648 (2004).

[34] C.A. Cerdeiriña, J. Troncoso, D. González-Salgado, G. García-Miaja, G.O. Hernández-Segura, D. Bessieres, M. Medeiros, L. Romaní, and M. Costas, J. Phys. Chem. B 111, 1119 (2007).

[35] F. Llovell and L.F. Vega, J. Phys. Chem. B 110, 11427 (2006).

[36] T. Lafitte, D. Bessieres, M.M. Piñeiro, and J.-L. Daridon, J. Chem. Phys. 124, 024509 (2006).

[37] T. Lafitte, M.M. Piñeiro, J.-L Daridon, and D. Bessieres, J. Phys. Chem. B 111, 3447 (2007).

[38] M. Medeiros and P. Téllez-Arredondo, Ind. Eng. Chem. Res. 47, 5723 (2008).

[39] R. Reynoso-López, P. Téllez-Arredondo, and M. Medeiros, Fluid Phase Equilib. 297, 98 (2010).

[40] P.G. Debenedetti, J. Phys.: Condens. Matter 15, R1669 (2003).

[41] S. Sastry, P.G. Debenedetti, F. Sciortino, and H.E. Stanley, Phys. Rev. E 53, 6144 (1996).

[42] L. P. N. Rebelo, P.G. Debenedetti, and S. Sastry, J. Chem. Phys. 109, 626 (1998). 


\section{FIGURE CAPTIONS}

Figure 1. Loci of extrema for the residual isochoric heat capacity $C_{V}^{\text {res }}$ of the LennardJones fluid from MBWR (A), PVE/hBH (B), and LJ-vdW (C) equations of state. Lines in (a) correspond to isothermal extrema, which, as indicated explicitly, divide the $p^{*}-T^{*}$ plane into regions of different sign of $\left(\partial C_{V}^{\text {res }} / \partial p\right)_{T}$; lines in (b) correspond to isobaric extrema, which delimitate regions of different sign of $\left(\partial C_{V}^{\text {res }} / \partial T\right)_{p} .(-)$ maxima; $(\cdots)$ minima. Data for the phase equilibrium lines were taken from Refs. 15-17 (liquid-gas) and Ref. 24 (solid-liquid). As defined in Section $2, p^{*} \equiv p \sigma^{3} / \varepsilon$ and $T^{*} \equiv T k_{B} / \varepsilon$.

Figure 2. Loci of extrema for the residual isobaric heat capacity $C_{p}^{r e s}$ of the LennardJones fluid from MBWR (A), PVE/hBH (B), and LJ-vdW (C) equations of state. Lines in (a) correspond to isothermal extrema, which, as indicated explicitly, divide the $p^{*}-T^{*}$ plane into regions of different sign of $\left(\partial C_{p}^{\text {res }} / \partial p\right)_{T}$; lines in (b) correspond to isobaric extrema, which delimitate regions of different sign of $\left(\partial C_{p}^{\text {res }} / \partial T\right)_{p} .(-)$ maxima; $(\cdots)$ minima. Data for the phase equilibrium lines were taken from Refs. 15-17 (liquid-gas) and Ref. 24 (solid-liquid). As defined in Section $2, p^{*} \equiv p \sigma^{3} / \varepsilon$ and $T^{*} \equiv T k_{B} / \varepsilon$.

Figure 3. Loci of extrema for the isobaric thermal expansivity $\alpha_{p}$ of the Lennard-Jones fluid from MBWR (A), PVE/hBH (B), and LJ-vdW (C) equations of state. Lines in (a) correspond to isothermal extrema, which, as indicated explicitly, divide the $p^{*}-T^{*}$ 
plane into regions of different sign of $\left(\partial \alpha_{p} / \partial p\right)_{T}$; lines in (b) correspond to isobaric extrema, which delimitate regions of different sign of $\left(\partial \alpha_{p} / \partial T\right)_{p} .(-)$ maxima; $(\cdots)$ minima. Data for the phase equilibrium lines were taken from Refs. 15-17 (liquid-gas) and Ref. 24 (solid-liquid). As defined in Section 2, $p^{*} \equiv p \sigma^{3} / \varepsilon$ and $T^{*} \equiv T k_{B} / \varepsilon$.

Figure 4. Loci of extrema for the isothermal compressibility $\kappa_{T}$ of the Lennard-Jones fluid from MBWR (A), PVE/hBH (B), and LJ-vdW (C) equations of state. Lines in (a) correspond to isothermal extrema, which, as indicated explicitly, divide the $p^{*}-T^{*}$ plane into regions of different sign of $\left(\partial \kappa_{T} / \partial p\right)_{T}$; lines in (b) correspond to isobaric extrema, which delimitate regions of different sign of $\left(\partial \kappa_{T} / \partial T\right)_{p} .(-)$ maxima; $(\cdots)$ minima. Data for the phase equilibrium lines were taken from Refs. 15-17 (liquid-gas) and Ref. 24 (solid-liquid). As defined in Section 2, $p^{*} \equiv p \sigma^{3} / \varepsilon$ and $T^{*} \equiv T k_{B} / \varepsilon$. 
Figure 1

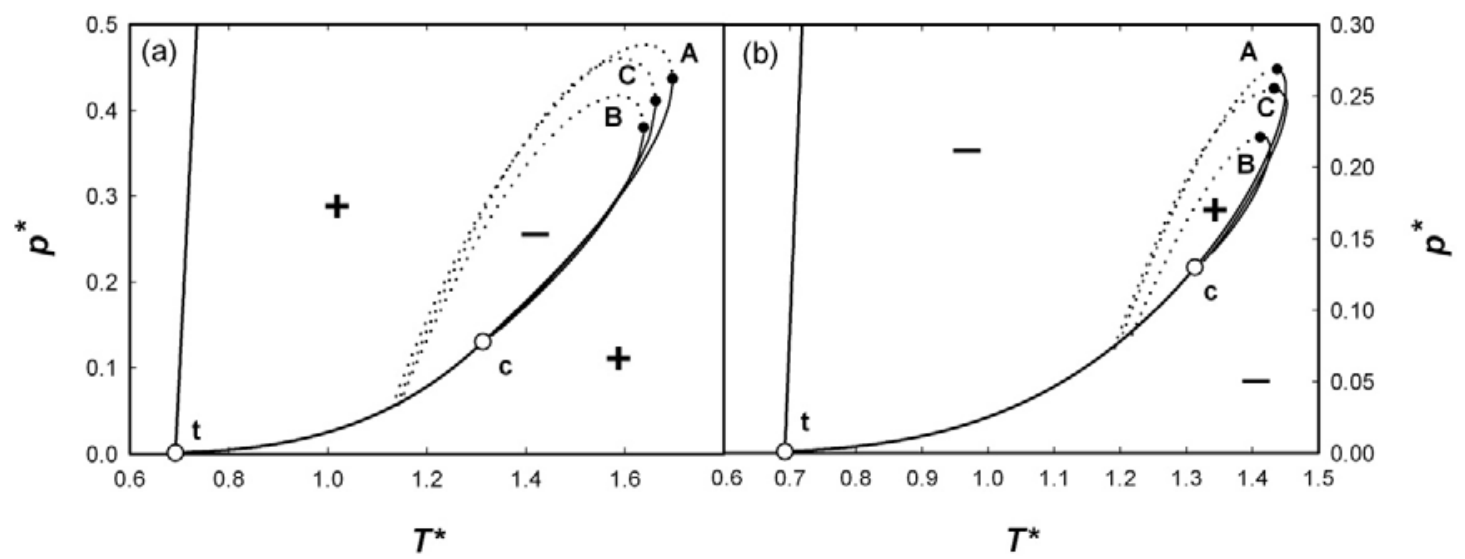


Figure 2

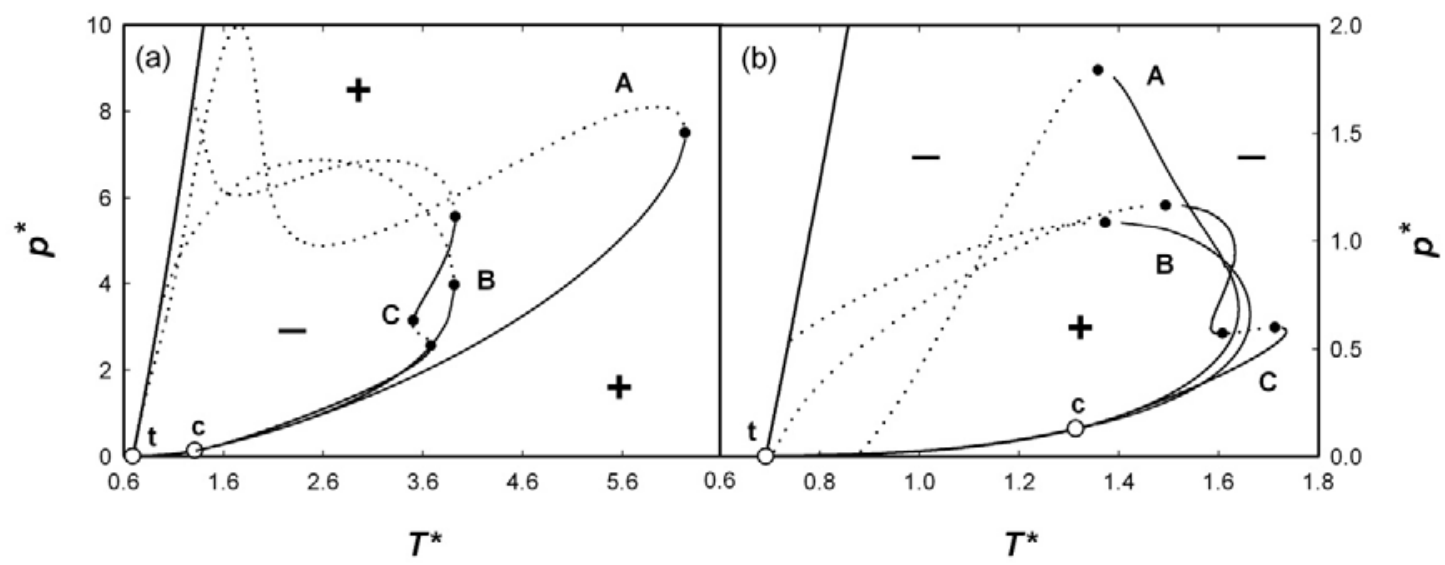

41 
Figure 3

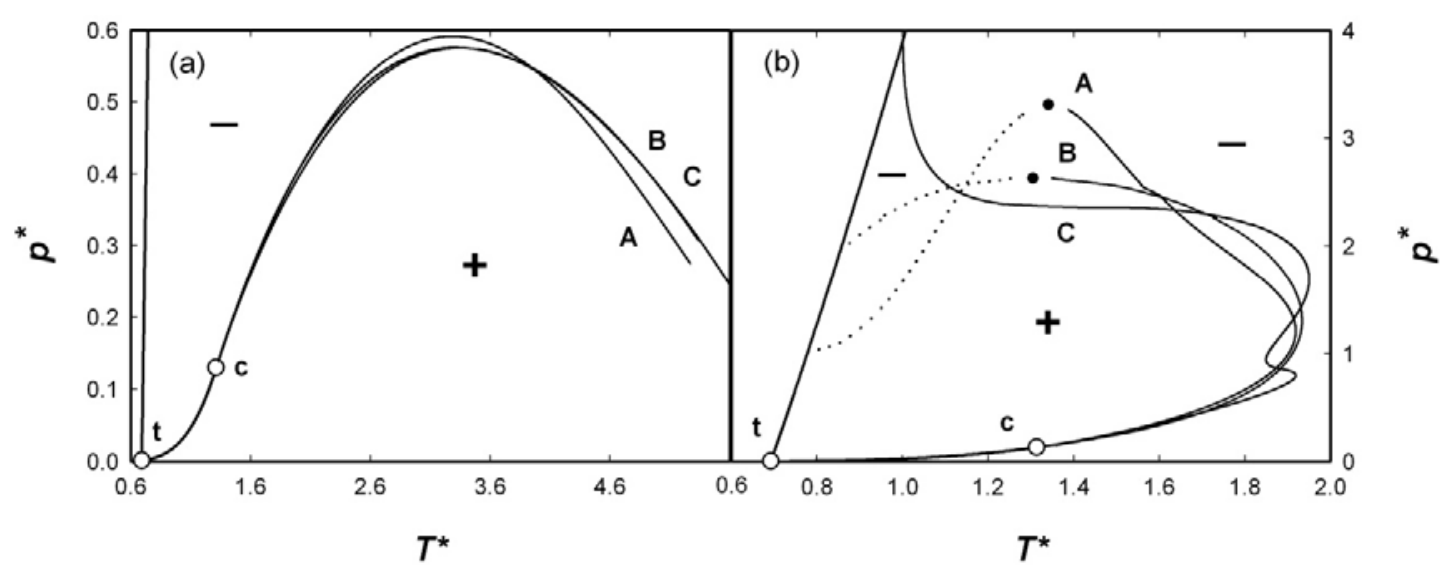


Figure 4

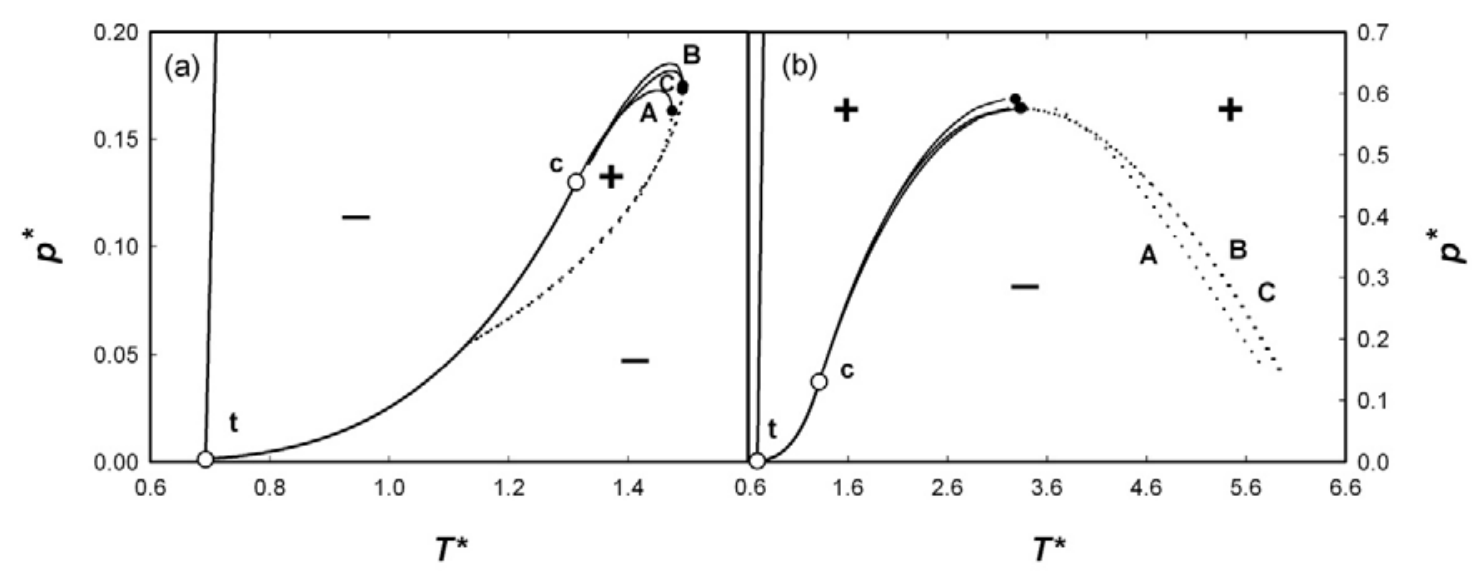

41 